\title{
Research Article \\ Fixed Points, Inner Product Spaces, and Functional Equations
}

\author{
Choonkil Park \\ Department of Mathematics, Research Institute for Natural Sciences, Hanyang University, \\ Seoul 133-791, Republic of Korea
}

Correspondence should be addressed to Choonkil Park, baak@hanyang.ac.kr

Received 1 February 2010; Revised 30 May 2010; Accepted 5 July 2010

Academic Editor: Marlène Frigon

Copyright ( $) 2010$ Choonkil Park. This is an open access article distributed under the Creative Commons Attribution License, which permits unrestricted use, distribution, and reproduction in any medium, provided the original work is properly cited.

Rassias introduced the following equality $\sum_{i, j=1}^{n}\left\|x_{i}-x_{j}\right\|^{2}=2 n \sum_{i=1}^{n}\left\|x_{i}\right\|^{2}, \sum_{i=1}^{n} x_{i}=0$, for a fixed integer $n \geq 3$. Let $V, W$ be real vector spaces. It is shown that, if a mapping $f: V \rightarrow W$ satisfies the following functional equation $\sum_{i, j=1}^{n} f\left(x_{i}-x_{j}\right)=2 n \sum_{i=1}^{n} f\left(x_{i}\right)$ for all $x_{1}, \ldots, x_{n} \in V$ with $\sum_{i=1}^{n} x_{i}=0$, which is defined by the above equality, then the mapping $f: V \rightarrow W$ is realized as the sum of an additive mapping and a quadratic mapping. Using the fixed point method, we prove the generalized Hyers-Ulam stability of the above functional equation in real Banach spaces.

\section{Introduction and Preliminaries}

The stability problem of functional equations originated from a question of Ulam [1] concerning the stability of group homomorphisms. Hyers [2] gave a first affirmative partial answer to the question of Ulam for Banach spaces. Hyers' theorem was generalized by Aoki [3] for additive mappings and by Rassias [4] for linear mappings by considering an unbounded Cauchy difference. The paper of Rassias [4] has provided a lot of influence on the development of what we call the generalized Hyers-Ulam stability or Hyers-Ulam-Rassias stability of functional equations. A generalization of the Rassias theorem was obtained by Găvruţa [5] by replacing the unbounded Cauchy difference by a general control function in the spirit of Rassias' approach.

A square norm on an inner product space satisfies the parallelogram equality

$$
\|x+y\|^{2}+\|x-y\|^{2}=2\|x\|^{2}+2\|y\|^{2} .
$$


The functional equation

$$
f(x+y)+f(x-y)=2 f(x)+2 f(y)
$$

is called a quadratic functional equation. A generalized Hyers-Ulam stability problem for the quadratic functional equation was proved by Skof [6] for mappings $f: X \rightarrow Y$, where $X$ is a normed space and $Y$ is a Banach space. Cholewa [7] noticed that the theorem of Skof is still true if the relevant domain $X$ is replaced by an Abelian group. Czerwik [8] proved the generalized Hyers-Ulam stability of the quadratic functional equation. The generalized Hyers-Ulam stability of the above quadratic functional equation and of two functional equations of quadratic type was obtained by Cădariu and Radu [9].

By a square norm on an inner product space, Rassias [10] introduced the following equality:

$$
\sum_{i, j=1}^{n}\left\|x_{i}-x_{j}\right\|^{2}=2 n \sum_{i=1}^{n}\left\|x_{i}\right\|^{2}, \quad \sum_{i=1}^{n} x_{i}=0,
$$

for a fixed integer $n \geq 3$. By the above equality, we can define the following functional equation:

$$
\sum_{i, j=1}^{n} f\left(x_{i}-x_{j}\right)=2 n \sum_{i=1}^{n} f\left(x_{i}\right)
$$

for all $x_{1}, \ldots, x_{n} \in V$ with $\sum_{i=1}^{n} x_{i}=0$, where $V$ is a real vector space.

A square norm on an inner product space satisfies

$$
\sum_{i, j=1}^{3}\left\|x_{i}-x_{j}\right\|^{2}=6 \sum_{i=1}^{3}\left\|x_{i}\right\|^{2}
$$

for all $x_{1}, x_{2}, x_{3} \in \mathbb{R}$ with $x_{1}+x_{2}+x_{3}=0$ (see [10]).

From the above equality we can define the following functional equation:

$$
h(x-y)+h(2 x+y)+h(x+2 y)=3 h(x)+3 h(y)+3 h(x+y)
$$

which is called a functional equation of quadratic type. In fact, $h(x)=a x^{2}$ in $\mathbb{R}$ satisfies the functional equation of quadratic type. In particular, every solution of the functional equation of quadratic type is said to be a quadratic-type mapping. One can easily show that if $h$ satisfies the quadratic functional equation then $h$ satisfies the functional equation of quadratic type. The stability problems of several functional equations have been extensively investigated by a number of authors, and there are many interesting results concerning this problem (see [11-24]). 
Let $X$ be a set. Then, a function $d: X \times X \rightarrow[0, \infty]$ is called a generalized metric on $X$ if $d$ satisfies the following:

(1) $d(x, y)=0$ if and only if $x=y$,

(2) $d(x, y)=d(y, x)$ for all $x, y \in X$,

(3) $d(x, z) \leq d(x, y)+d(y, z)$ for all $x, y, z \in X$.

We recall a fundamental result in fixed point theory.

Theorem 1.1 (see $[25,26])$. Let $(X, d)$ be a complete generalized metric space, and let $J: X \rightarrow X$ be a strictly contractive mapping with the, Lipschitz constant $L<1$. Then, for each given element $x \in X$, either

$$
d\left(J^{n} x, J^{n+1} x\right)=\infty
$$

for all nonnegative integers $n$ or there exists a positive integer $n_{0}$ such that

(1) $d\left(J^{n} x, J^{n+1} x\right)<\infty$, for all $n \geq n_{0}$,

(2) the sequence $\left\{J^{n} x\right\}$ converges to a fixed point $y^{*}$ of $J$,

(3) $y^{*}$ is the unique fixed point of $J$ in the set $Y=\left\{y \in X \mid d\left(J^{n_{0}} x, y\right)<\infty\right\}$,

(4) $d\left(y, y^{*}\right) \leq(1 /(1-L)) d(y, J y)$ for all $y \in Y$.

In 1996, Isac and Rassias [27] were the first to provide applications of stability theory of functional equations for the proof of new fixed point theorems with applications. By using the fixed point method, the stability problems of several functional equations have been extensively investigated by a number of authors (see [17, 28-31]).

Throughout this paper, assume that $n$ is a fixed integer greater than 2. Let $X$ be a real normed vector space with norm $\|\cdot\|$, and let $Y$ be a real Banach space with norm $\|\cdot\|$.

In this paper, we investigate the functional equation (1.4). Using the fixed point method, we prove the generalized Hyers-Ulam stability of the functional equation (1.4) in real Banach spaces.

\section{Fixed Points and Functional Equations Associated with Inner Product Spaces}

We investigate the functional equation (1.4).

Lemma 2.1. Let $V$ and $W$ be real vector spaces. If a mapping $f: V \rightarrow W$ satisfies

$$
\sum_{i, j=1}^{n} f\left(x_{i}-x_{j}\right)=2 n \sum_{i=1}^{n} f\left(x_{i}\right)
$$

for all $x_{1}, \ldots, x_{n} \in V$ with $\sum_{i=1}^{n} x_{i}=0$, then the mapping $f: V \rightarrow W$ is realized as the sum of an additive mapping and a quadratic-type mapping. 
Proof. Let $g(x):=(f(x)-f(-x)) / 2$ and $h(x):=(f(x)+f(-x)) / 2$ for all $x \in V$. Then, $g(x)$ is an odd mapping and $h(x)$ is an even mapping satisfying $f(x)=g(x)+h(x)$ and (2.1). we get

Letting $x_{1}=x, x_{2}=y, x_{3}=-x-y$, and $x_{4}=\cdots=x_{n}=0$ in (2.1) for the mapping $g$,

$$
g(x)+g(y)-g(x+y)=g(x)+g(y)+g(-x-y)=0
$$

for all $x, y \in V$. So, $g(x)$ is an additive mapping.

Letting $x_{1}=x, x_{2}=y, x_{3}=-x-y$, and $x_{4}=\cdots=x_{n}=0$ in (2.1) for the mapping $h$, we get

$$
h(x-y)+h(2 x+y)+h(x+2 y)=3 h(x)+3 h(y)+3 h(x+y)
$$

for all $x, y \in V$. So, $h(x)$ is a quadratic-type mapping.

For a given mapping $f: X \rightarrow Y$, we define

$$
D f\left(x_{1}, \ldots, x_{n}\right):=\sum_{i, j=1}^{n} f\left(x_{i}-x_{j}\right)-2 n \sum_{i=1}^{n} f\left(x_{i}\right)
$$

for all $x_{1}, \ldots, x_{n} \in X$.

Let $g(x):=(f(x)-f(-x)) / 2$ and $h(x):=(f(x)+f(-x)) / 2$ for all $x \in X$. Then, $g(x)$ is an odd mapping and $h(x)$ is an even mapping satisfying $f(x)=g(x)+h(x)$. If $D f\left(x_{1}, \ldots, x_{n}\right)=0$, then $\operatorname{Dg}\left(x_{1}, \ldots, x_{n}\right)=0$ and $\operatorname{Dh}\left(x_{1}, \ldots, x_{n}\right)=0$.

Using the fixed point method, we prove the generalized Hyers-Ulam stability of the functional equation $D f\left(x_{1}, \ldots, x_{n}\right)=0$ in real Banach spaces.

Theorem 2.2. Let $f: X \rightarrow Y$ be a mapping with $f(0)=0$ for which there exists a function $\varphi: X^{n} \rightarrow[0, \infty)$ such that there exists an $L<1$ such that

$$
\begin{gathered}
\varphi\left(x_{1}, \ldots, x_{n}\right) \leq \frac{L}{4} \varphi\left(2 x_{1}, \ldots, 2 x_{n}\right), \\
\left\|D h\left(x_{1}, \ldots, x_{n}\right)\right\| \leq \varphi\left(x_{1}, \ldots, x_{n}\right)
\end{gathered}
$$

for all $x_{1}, \ldots, x_{n} \in X$ with $\sum_{i=1}^{n} x_{i}=0$. Then, there exists a unique quadratic-type mapping $Q: X \rightarrow$ $Y$ satisfying

$$
\|h(x)-Q(x)\| \leq \frac{L}{8-8 L} \varphi(x,-x, \underbrace{0, \ldots, 0}_{n-2 \text { times }})
$$

for all $x \in X$.

Proof. Consider the set

$$
S:=\{\psi: X \longrightarrow Y\},
$$


Fixed Point Theory and Applications

and introduce the generalized metric on $S$ :

$$
d\left(\psi_{1}, \psi_{2}\right)=\inf \left\{K \in \mathbb{R}_{+}:\left\|\psi_{1}(x)-\psi_{2}(x)\right\| \leq K \varphi(x,-x, \underbrace{0, \ldots, 0}_{n-2 \text { times }}), \forall x \in X\right\} .
$$

By the same method given in $[17,28,32]$, one can easily show that $(S, d)$ is complete.

Now we consider the linear mapping $J: S \rightarrow S$ such that

$$
J \psi(x):=4 \psi\left(\frac{x}{2}\right)
$$

for all $x \in X$.

It follows from the proof of Theorem 3.1 of [25] that

$$
d\left(J \psi_{1}, J \psi_{2}\right) \leq L d\left(\psi_{1}, \psi_{2}\right)
$$

for all $\psi_{1}, \psi_{2} \in S$.

Letting $x_{1}=x, x_{2}=-x$, and $x_{3}=\cdots=x_{n}=0$ in (2.6), we get

$$
\|2 h(2 x)-8 h(x)\| \leq \varphi(x,-x, \underbrace{0, \ldots, 0}_{n-2 \text { times }})
$$

for all $x \in X$. It follows from (2.12) that

$$
\begin{aligned}
\left\|h(x)-4 h\left(\frac{x}{2}\right)\right\| & \leq \frac{1}{2} \varphi(\frac{x}{2},-\frac{x}{2}, \underbrace{0, \ldots, 0}_{n-2 \text { times }}) \\
& \leq \frac{L}{8} \varphi(x,-x, \underbrace{0, \ldots, 0}_{n-2 \text { times }})
\end{aligned}
$$

for all $x \in X$. Hence, $d(h, J h) \leq L / 8$.

By Theorem 1.1, there exists a mapping $Q: X \rightarrow Y$ satisfying the following.

(1) $Q$ is a fixed point of $J$; that is,

$$
Q\left(\frac{x}{2}\right)=\frac{1}{4} Q(x)
$$

for all $x \in X$. The mapping $Q$ is a unique fixed point of $J$ in the set

$$
M=\{\psi \in S: d(\psi, h)<\infty\}
$$


This implies that $Q$ is a unique mapping satisfying (2.14) such that there exists a $K \in(0, \infty)$ satisfying

$$
\|h(x)-Q(x)\| \leq K \varphi(x,-x, \underbrace{0, \ldots, 0}_{n-2 \text { times }})
$$

for all $x \in X$.

(2) One has $d\left(J^{m} h, Q\right) \rightarrow 0$ as $m \rightarrow \infty$. This implies the equality

$$
\lim _{d \rightarrow \infty} 4^{d} h\left(\frac{x}{2^{d}}\right)=Q(x)
$$

for all $x \in X$. Since $h$ is an even mapping, $Q: X \rightarrow Y$ is an even mapping.

Moreover,one has $(3) d(h, Q) \leq(1 /(1-L)) d(h, J h)$, which implies the inequality

$$
d(h, Q) \leq \frac{L}{8-8 L}
$$

This implies that inequality (2.7) holds.

It follows from (2.5), (2.6), and (2.17) that

$$
\begin{aligned}
\left\|D Q\left(x_{1}, \ldots, x_{n}\right)\right\| & =\lim _{d \rightarrow \infty} 4^{d}\left\|D h\left(\frac{x_{1}}{2^{d}}, \ldots, \frac{x_{n}}{2^{d}}\right)\right\| \\
& \leq \lim _{d \rightarrow \infty} 4^{d} \varphi\left(\frac{x_{1}}{2^{d}}, \ldots, \frac{x_{n}}{2^{d}}\right)=0
\end{aligned}
$$

for all $x_{1}, \ldots, x_{n} \in X$ with $\sum_{i=1}^{n} x_{i}=0$. So, $D Q\left(x_{1}, \ldots, x_{n}\right)=0$ for all $x_{1}, \ldots, x_{n} \in X$ with $\sum_{i=1}^{n} x_{i}=0$. By Lemma 2.1, the mapping $Q: X \rightarrow Y$ is a quadratic-type mapping.

Therefore, there exists a unique quadratic-type mapping $Q: X \rightarrow Y$ satisfying (2.7).

Corollary 2.3. Let $p>2$ and $\theta \geq 0$ be real numbers, and let $f: X \rightarrow Y$ be a mapping such that

$$
\left\|D h\left(x_{1}, \ldots, x_{n}\right)\right\| \leq \theta \sum_{j=1}^{n}\left\|x_{j}\right\|^{p}
$$

for all $x_{1}, \ldots, x_{n} \in X$ with $\sum_{i=1}^{n} x_{i}=0$. Then, there exists a unique quadratic-type mapping $Q: X \rightarrow$ $Y$ satisfying

$$
\|h(x)-Q(x)\| \leq \frac{\theta}{2^{p}-4}\|x\|^{p}
$$

for all $x \in X$. 
Proof. The proof follows from Theorem 2.2 by taking

$$
\varphi\left(x_{1}, \ldots, x_{n}\right):=\theta \sum_{j=1}^{n}\left\|x_{j}\right\|^{p}
$$

for all $x_{1}, \ldots, x_{n} \in X$. Then, we can choose $L=2^{2-p}$, and we get the desired result.

Remark 2.4. Let $f: X \rightarrow Y$ be a mapping for which there exists a function $\varphi: X^{n} \rightarrow[0, \infty)$ satisfying (2.6) and $f(0)=0$ such that there exists an $L<1$ such that

$$
\varphi\left(x_{1}, \ldots, x_{n}\right) \leq 4 L \varphi\left(\frac{x_{1}}{2}, \ldots, \frac{x_{n}}{2}\right)
$$

for all $x_{1}, \ldots, x_{n} \in X$ with $\sum_{i=1}^{n} x_{i}=0$. By a similar method to the proof of Theorem 2.2, one can show that there exists a unique quadratic-type mapping $Q: X \rightarrow Y$ satisfying

$$
\|h(x)-Q(x)\| \leq \frac{1}{8-8 L} \varphi(x,-x, \underbrace{0, \ldots, 0}_{n-2 \text { times }})
$$

for all $x \in X$.

For the case $p<2$, one can obtain a similar result to Corollary 2.3: let $p<2$ and $\theta$ be positive real numbers, and let $f: X \rightarrow Y$ be a mapping satisfying (2.20). Then, there exists a unique quadratic-type mapping $Q: X \rightarrow Y$ satisfying

$$
\|h(x)-Q(x)\| \leq \frac{\theta}{4-2^{p}}\|x\|^{p}
$$

for all $x \in X$.

Theorem 2.5. Let $f: X \rightarrow Y$ be a mapping for which there exists a function $\varphi: X^{n} \rightarrow[0, \infty)$ such that there exists an $L<1$ such that

$$
\begin{aligned}
& \varphi\left(x_{1}, \ldots, x_{n}\right) \leq \frac{L}{2} \varphi\left(2 x_{1}, \ldots, 2 x_{n}\right), \\
& \left\|D g\left(x_{1}, \ldots, x_{n}\right)\right\| \leq \varphi\left(x_{1}, \ldots, x_{n}\right)
\end{aligned}
$$

for all $x_{1}, \ldots, x_{n} \in X$ with $\sum_{i=1}^{n} x_{i}=0$. Then, there exists a unique additive mapping $A: X \rightarrow Y$ satisfying

$$
\|g(x)-A(x)\| \leq \frac{L}{4 n-4 n L} \varphi(x, x,-2 x, \underbrace{0, \ldots, 0}_{n-3 \text { times }})
$$

for all $x \in X$. 
Proof. Consider the set

$$
S:=\{\psi: X \longrightarrow Y\},
$$

and introduce the generalized metric on $S$ :

$$
d\left(\psi_{1}, \psi_{2}\right)=\inf \left\{K \in \mathbb{R}_{+}:\left\|\psi_{1}(x)-\psi_{2}(x)\right\| \leq K \varphi(x, x,-2 x, \underbrace{0, \ldots, 0}_{n-3 \text { times }}), \forall x \in X\right\} .
$$

By the same method given in $[17,28,32]$, one can easily show that $(S, d)$ is complete.

Now we consider the linear mapping $J: S \rightarrow S$ such that

$$
J \psi(x):=2 \psi\left(\frac{x}{2}\right)
$$

for all $x \in X$.

It follows from the proof of Theorem 3.1 of [25] that

$$
d\left(J \psi_{1}, J \psi_{2}\right) \leq L d\left(\psi_{1}, \psi_{2}\right)
$$

for all $\psi_{1}, \psi_{2} \in S$.

Letting $x_{1}=x_{2}=x$ and $x_{3}=\cdots=x_{n}=0$ in (2.27), we get

$$
\|4 n g(x)-2 n g(2 x)\| \leq \varphi(x, x,-2 x, \underbrace{0, \ldots, 0}_{n-3 \text { times }})
$$

for all $x \in X$. It follows from (2.33) that

$$
\begin{aligned}
\left\|g(x)-2 g\left(\frac{x}{2}\right)\right\| & \leq \frac{1}{2 n} \varphi(\frac{x}{2}, \frac{x}{2},-x, \underbrace{0, \ldots, 0}_{n-3 \text { times }}) \\
& \leq \frac{L}{4 n} \varphi(x, x,-2 x, \underbrace{0, \ldots, 0}_{n-3 \text { times }})
\end{aligned}
$$

for all $x \in X$. Hence, $d(g, J g) \leq L / 4 n$. 
Fixed Point Theory and Applications

By Theorem 1.1, there exists a mapping $A: X \rightarrow Y$ satisfying the following.

(1) $A$ is a fixed point of $J$; that is,

$$
A\left(\frac{x}{2}\right)=\frac{1}{2} A(x)
$$

for all $x \in X$. The mapping $A$ is a unique fixed point of $J$ in the set

$$
M=\{\psi \in S: d(\psi, g)<\infty\}
$$

This implies that $A$ is a unique mapping satisfying (2.35) such that there exists a $K \in(0, \infty)$ satisfying

$$
\|g(x)-A(x)\| \leq K \varphi(x, x,-2 x, \underbrace{0, \ldots, 0}_{n-3 \text { times }})
$$

for all $x \in X$.

(2) One has $d\left(J^{m} g, A\right) \rightarrow 0$ as $m \rightarrow \infty$. This implies the equality

$$
\lim _{d \rightarrow \infty} 2^{d} g\left(\frac{x}{2^{d}}\right)=A(x)
$$

for all $x \in X$. Since $g$ is an odd mapping, $A: X \rightarrow Y$ is an odd mapping;

(3) Moreover $d(g, A) \leq(1 /(1-L)) d(g, J g)$, which implies the inequality

$$
d(g, A) \leq \frac{L}{4 n-4 n L}
$$

This implies that inequality (2.28) holds.

It follows from (2.26), (2.27), and (2.38) that

$$
\begin{aligned}
\left\|D A\left(x_{1}, \ldots, x_{n}\right)\right\| & =\lim _{d \rightarrow \infty} n^{d}\left\|D g\left(\frac{x_{1}}{2^{d}}, \ldots, \frac{x_{n}}{2^{d}}\right)\right\| \\
& \leq \lim _{d \rightarrow \infty} 2^{d} \varphi\left(\frac{x_{1}}{2^{d}}, \ldots, \frac{x_{n}}{2^{d}}\right)=0
\end{aligned}
$$

for all $x_{1}, \ldots, x_{n} \in X$ with $\sum_{i=1}^{n} x_{i}=0$. So, $D A\left(x_{1}, \ldots, x_{n}\right)=0$ for all $x_{1}, \ldots, x_{n} \in X$ with $\sum_{i=1}^{n} x_{i}=0$. By Lemma 2.1, the mapping $A: X \rightarrow Y$ is an additive mapping. desired.

Therefore, there exists a unique additive mapping $A: X \rightarrow Y$ satisfying (2.28), as 
Corollary 2.6. Let $p>1$ and $\theta \geq 0$ be real numbers, and let $f: X \rightarrow Y$ be a mapping satisfying (2.20). Then, there exists a unique additive mapping $A: X \rightarrow Y$ satisfying

$$
\|g(x)-A(x)\| \leq \frac{\left(2^{p}+2\right) \theta}{2 n\left(2^{p}-2\right)}\|x\|^{p}
$$

for all $x \in X$.

Proof. The proof follows from Theorem 2.5 by taking

$$
\varphi\left(x_{1}, \ldots, x_{n}\right):=\theta \sum_{j=1}^{n}\left\|x_{j}\right\|^{p}
$$

for all $x_{1}, \ldots, x_{n} \in X$. Then, we can choose $L=2^{1-p}$, and we get the desired result.

Remark 2.7. Let $f: X \rightarrow Y$ be a mapping for which there exists a function $\varphi: X^{n} \rightarrow[0, \infty)$ satisfying (2.27) such that there exists an $L<1$ such that

$$
\varphi\left(x_{1}, \ldots, x_{n}\right) \leq 2 L \varphi\left(\frac{x_{1}}{2}, \ldots, \frac{x_{n}}{2}\right)
$$

for all $x_{1}, \ldots, x_{n} \in X$. By a similar method to the proof of Theorem 2.5, one can show that there exists a unique additive mapping $A: X \rightarrow Y$ satisfying

$$
\|g(x)-A(x)\| \leq \frac{1}{4 n-4 n L} \varphi(x, x,-2 x, \underbrace{0, \ldots, 0}_{n-3 \text { times }})
$$

for all $x \in X$.

For the case $p<1$, one can obtain a similar result to Corollary 2.6: let $p<1$ and $\theta$ be positive real numbers, and let $f: X \rightarrow Y$ be a mapping satisfying (2.20). Then, there exists a unique additive mapping $A: X \rightarrow Y$ satisfying

$$
\|g(x)-A(x)\| \leq \frac{\left(2+2^{p}\right) \theta}{2 n\left(2-2^{p}\right)}\|x\|^{p}
$$

for all $x \in X$.

Since

$$
\begin{gathered}
\left\|D f\left(x_{1}, \ldots, x_{n}\right)\right\| \leq \varphi\left(x_{1}, \ldots, x_{n}\right), \\
\left\|D h\left(x_{1}, \ldots, x_{n}\right)\right\| \leq \frac{1}{2} \varphi\left(x_{1}, \ldots, x_{n}\right)+\frac{1}{2} \varphi\left(-x_{1}, \ldots,-x_{n}\right), \\
\left\|D g\left(x_{1}, \ldots, x_{n}\right)\right\| \leq \frac{1}{2} \varphi\left(x_{1}, \ldots, x_{n}\right)+\frac{1}{2} \varphi\left(-x_{1}, \ldots,-x_{n}\right) .
\end{gathered}
$$


Fixed Point Theory and Applications

Note that

$$
\frac{L}{4} \varphi\left(2 x_{1}, \ldots, 2 x_{n}\right) \leq \frac{L}{2} \varphi\left(2 x_{1}, \ldots, 2 x_{n}\right) .
$$

Combining Theorems 2.2 and 2.5, we obtain the following result.

Theorem 2.8. Let $f: X \rightarrow Y$ be a mapping satisfying $f(0)=0$ for which there exists a function $\varphi: X^{n} \rightarrow[0, \infty)$ satisfying $(2.5)$ and

$$
\left\|D f\left(x_{1}, \ldots, x_{n}\right)\right\| \leq \varphi\left(x_{1}, \ldots, x_{n}\right)
$$

for all $x_{1}, \ldots, x_{n} \in X$ with $\sum_{i=1}^{n} x_{i}=0$. Then, there exist an additive mapping $A: X \rightarrow Y$ and $a$ quadratic type mapping $Q: X \rightarrow Y$ such that

$$
\begin{aligned}
\|f(x)-A(x)-Q(x)\| \leq & \frac{L}{16-16 L}(\varphi(x,-x, \underbrace{0, \ldots, 0}_{n-2 \text { times }})+\varphi(-x, x, \underbrace{0, \ldots, 0}_{n-2 \text { times }})) \\
& +\frac{L}{8 n-8 n L}(\varphi(x, x,-2 x, \underbrace{0, \ldots, 0}_{n-3 \text { times }})+\varphi(-x,-x, 2 x, \underbrace{0, \ldots, 0}_{n-3 \text { times }}))
\end{aligned}
$$

for all $x \in X$.

Corollary 2.9. Let $p>2$ and $\theta$ be positive real numbers, and let $f: X \rightarrow Y$ be a mapping such that

$$
\left\|D f\left(x_{1}, \ldots, x_{n}\right)\right\| \leq \theta \sum_{i=1}^{n}\left\|x_{i}\right\|^{p}
$$

for all $x_{1}, \ldots, x_{n} \in X$ with $\sum_{i=1}^{n} x_{i}=0$. Then, there exist an additive mapping $A: X \rightarrow Y$ and $a$ quadratic-type mapping $Q: X \rightarrow Y$ such that

$$
\|f(x)-A(x)-Q(x)\| \leq\left(\frac{1}{2^{p}-4}+\frac{2^{p}+2}{2 n\left(2^{p}-2\right)}\right) \theta\|x\|^{p}
$$

for all $x \in X$. 
Proof. Define $\varphi\left(x_{1}, \ldots, x_{n}\right)=\theta \sum_{i=1}^{n}\left\|x_{i}\right\|^{p}$, and apply Theorem 2.8 to get the desired result.

Note that

$$
2 L \varphi\left(\frac{x_{1}}{2}, \ldots, \frac{x_{n}}{2}\right) \leq 4 L \varphi\left(\frac{x_{1}}{2}, \ldots, \frac{x_{n}}{2}\right) .
$$

Combining Remarks 2.4 and 2.7, we obtain the following result.

Remark 2.10. Let $f: X \rightarrow Y$ be a mapping for which there exists a function $\varphi: X^{n} \rightarrow[0, \infty)$ satisfying (2.48) and $f(0)=0$ such that there exists an $L<1$ such that

$$
\varphi\left(x_{1}, \ldots, x_{n}\right) \leq 2 L \varphi\left(\frac{x_{1}}{2}, \ldots, \frac{x_{n}}{2}\right)
$$

for all $x_{1}, \ldots, x_{n} \in X$. By a similar method to the proof of Theorem 2.8, one can show that there exist an additive mapping $A: X \rightarrow Y$ and a quadratic-type mapping $Q: X \rightarrow Y$ such that

$$
\begin{aligned}
\|f(x)-A(x)-Q(x)\| \leq & \frac{1}{16-16 L}(\varphi(x,-x, \underbrace{0, \ldots, 0}_{n-2 \text { times }})+\varphi(-x, x, \underbrace{0, \ldots, 0}_{n-2 \text { times }})) \\
& +\frac{1}{8 n-8 n L}(\varphi(x, x,-2 x, \underbrace{0, \ldots, 0}_{n-3 \text { times }})+\varphi(-x,-x, 2 x, \underbrace{0, \ldots, 0}_{n-3 \text { times }}))
\end{aligned}
$$

for all $x \in X$.

For the case $p<1$, one can obtain a similar result to Corollary 2.9: let $p<1$ and $\theta$ be positive real numbers, and let $f: X \rightarrow Y$ be a mapping satisfying (2.50). Then, there exist an additive mapping $A: X \rightarrow Y$ and a quadratic-type mapping $Q: X \rightarrow Y$ satisfying

$$
\|f(x)-A(x)-Q(x)\| \leq\left(\frac{1}{4-2^{p}}+\frac{2+2^{p}}{2 n\left(2-2^{p}\right)}\right) \theta\|x\|^{p}
$$

for all $x \in X$.

\section{Acknowledgment}

This paper was supported by Basic Science Research Program through the National Research Foundation of Korea funded by the Ministry of Education, Science and Technology (NRF2009-0070788). 


\section{References}

[1] S. M. Ulam, Problems in Modern Mathematics, John Wiley \& Sons, New York, NY, USA, 1960.

[2] D. H. Hyers, "On the stability of the linear functional equation," Proceedings of the National Academy of Sciences of the United States of America, vol. 27, pp. 222-224, 1941.

[3] T. Aoki, "On the stability of the linear transformation in Banach spaces," Journal of the Mathematical Society of Japan, vol. 2, pp. 64-66, 1950.

[4] Th. M. Rassias, "On the stability of the linear mapping in Banach spaces," Proceedings of the American Mathematical Society, vol. 72, no. 2, pp. 297-300, 1978.

[5] P. Găvruța, "A generalization of the Hyers-Ulam-Rassias stability of approximately additive mappings," Journal of Mathematical Analysis and Applications, vol. 184, no. 3, pp. 431-436, 1994.

[6] F. Skof, "Proprietà locali e approssimazione di operatori," Rendiconti del Seminario Matematico e Fisico di Milano, vol. 53, no. 1, pp. 113-129, 1983.

[7] P. W. Cholewa, "Remarks on the stability of functional equations," Aequationes Mathematicae, vol. 27, no. 1-2, pp. 76-86, 1984.

[8] S. Czerwik, "On the stability of the quadratic mapping in normed spaces," Abhandlungen aus dem Mathematischen Seminar der Universität Hamburg, vol. 62, pp. 59-64, 1992.

[9] L. Cădariu and V. Radu, "Fixed points and the stability of quadratic functional equations," Analele Universităţii de Vest din Timişoara, vol. 41, no. 1, pp. 25-48, 2003.

[10] Th. M. Rassias, "On characterizations of inner product spaces and generalizations of the H. Bohr inequality," in Topics in Mathematical Analysis, Th. M. Rassias, Ed., vol. 11, pp. 803-819, World Scientific, Teaneck, NJ, USA, 1989.

[11] D. H. Hyers, G. Isac, and Th. M. Rassias, Stability of Functional Equations in Several Variables, Progress in Nonlinear Differential Equations and Their Applications, 34, Birkhäuser, Boston, Mass, USA, 1998.

[12] M. S. Moslehian, "On the orthogonal stability of the Pexiderized quadratic equation," Journal of Difference Equations and Applications, vol. 11, no. 11, pp. 999-1004, 2005.

[13] C.-G. Park, "Homomorphisms between Poisson JC*-algebras," Bulletin of the Brazilian Mathematical Society, vol. 36, no. 1, pp. 79-97, 2005.

[14] C. Park, Y. S. Cho, and M.-H. Han, "Functional inequalities associated with Jordan-von Neumanntype additive functional equations," Journal of Inequalities and Applications, vol. 2007, Article ID 41820, 13 pages, 2007.

[15] C. Park and J. Cui, "Generalized stability of $C^{*}$-ternary quadratic mappings," Abstract and Applied Analysis, vol. 2007, Article ID 23282, 6 pages, 2007.

[16] C. Park and A. Najati, "Homomorphisms and derivations in $C^{*}$-algebras," Abstract and Applied Analysis, vol. 2007, Article ID 80630, 12 pages, 2007.

[17] V. Radu, "The fixed point alternative and the stability of functional equations," Fixed Point Theory, vol. 4, no. 1, pp. 91-96, 2003.

[18] Th. M. Rassias, "Problem 16; 2, Report of the 27th International Symp. on Functional Equations," Aequationes Mathematicae, vol. 39, pp. 292-293; 309, 1990.

[19] Th. M. Rassias, "On the stability of the quadratic functional equation and its applications," Studia Universitatis Babeş-Bolyai. Mathematica, vol. 43, no. 3, pp. 89-124, 1998.

[20] Th. M. Rassias, "The problem of S. M. Ulam for approximately multiplicative mappings," Journal of Mathematical Analysis and Applications, vol. 246, no. 2, pp. 352-378, 2000.

[21] Th. M. Rassias, "On the stability of functional equations in Banach spaces," Journal of Mathematical Analysis and Applications, vol. 251, no. 1, pp. 264-284, 2000.

[22] Th. M. Rassias, "On the stability of functional equations and a problem of Ulam," Acta Applicandae Mathematicae, vol. 62, no. 1, pp. 23-130, 2000.

[23] Th. M. Rassias and P. Šemrl, "On the Hyers-Ulam stability of linear mappings," Journal of Mathematical Analysis and Applications, vol. 173, no. 2, pp. 325-338, 1993.

[24] Th. M. Rassias and K. Shibata, "Variational problem of some quadratic functionals in complex analysis," Journal of Mathematical Analysis and Applications, vol. 228, no. 1, pp. 234-253, 1998.

[25] L. Cădariu and V. Radu, "Fixed points and the stability of Jensen's functional equation," Journal of Inequalities in Pure and Applied Mathematics, vol. 4, no. 1, article 4, 2003.

[26] J. B. Diaz and B. Margolis, "A fixed point theorem of the alternative, for contractions on a generalized complete metric space," Bulletin of the American Mathematical Society, vol. 74, pp. 305-309, 1968. 
[27] G. Isac and Th. M. Rassias, "Stability of $\psi$-additive mappings: applications to nonlinear analysis," International Journal of Mathematics and Mathematical Sciences, vol. 19, no. 2, pp. 219-228, 1996.

[28] L. Cădariu and V. Radu, "Fixed point methods for the generalized stability of functional equations in a single variable," Fixed Point Theory and Applications, vol. 2008, Article ID 749392, 15 pages, 2008.

[29] M. Mirzavaziri and M. S. Moslehian, "A fixed point approach to stability of a quadratic equation," Bulletin of the Brazilian Mathematical Society, vol. 37, no. 3, pp. 361-376, 2006.

[30] C. Park, "Fixed points and Hyers-Ulam-Rassias stability of Cauchy-Jensen functional equations in Banach algebras," Fixed Point Theory and Applications, vol. 2007, Article ID 50175, 15 pages, 2007.

[31] C. Park, "Generalized Hyers-Ulam stability of quadratic functional equations: a fixed point approach," Fixed Point Theory and Applications, vol. 2008, Article ID 493751, 9 pages, 2008.

[32] L. Cădariu and V. Radu, "On the stability of the Cauchy functional equation: a fixed point approach," in Iteration Theory (ECIT '02), vol. 346 of Grazer Mathematische Berichte, pp. 43-52, Karl-FranzensUniversitaet Graz, Graz, Austria, 2004. 